\title{
Impaired Rho GTPase activation abrogates cell polarization and migration in macrophages with defective lipolysis
}

\author{
Elma Aflaki · Nariman A. B. Balenga $\cdot$ Petra Luschnig-Schratl $\cdot$ Heimo Wolinski • \\ Silvia Povoden • Prakash G. Chandak · Juliane G. Bogner-Strauss · Sandra Eder • \\ Viktoria Konya $\cdot$ Sepp-Dieter Kohlwein · Akos Heinemann · Dagmar Kratky
}

Received: 28 December 2010/Revised: 22 March 2011/Accepted: 7 April 2011/Published online: 2 May 2011

(C) The Author(s) 2011. This article is published with open access at Springerlink.com

\begin{abstract}
Infiltration of monocytes and macrophages into the site of inflammation is critical in the progression of inflammatory diseases such as atherosclerosis. Cell migration is dependent on the continuous organization of the actin cytoskeleton, which is regulated by members of the small Rho GTPase family (RhoA, Cdc42, Rac) that are also important for the regulation of signal transduction pathways. We have recently reported on reduced plaque formation in an atherosclerotic mouse model transplanted with bone marrow from adipose triglyceride lipase-deficient $(A t g l-/-)$ mice. Here we provide evidence that defective lipolysis in macrophages lacking ATGL, the major enzyme responsible for triacylglycerol hydrolysis, favors an anti-inflammatory M2-like macrophage
\end{abstract}

Electronic supplementary material The online version of this article (doi:10.1007/s00018-011-0688-4) contains supplementary material, which is available to authorized users.

E. Aflaki · S. Povoden $\cdot$ P. G. Chandak $\cdot$ D. Kratky $(\bowtie)$

Institute of Molecular Biology and Biochemistry,

Center of Molecular Medicine, Medical University of Graz,

Harrachgasse 21, 8010 Graz, Austria

e-mail: dagmar.kratky@medunigraz.at

N. A. B. Balenga $\cdot$ P. Luschnig-Schratl · V. Konya ·

A. Heinemann

Institute of Experimental and Clinical Pharmacology,

Medical University of Graz, Universitätsplatz 4,

8010 Graz, Austria

H. Wolinski · S. Eder · S.-D. Kohlwein

Institute of Molecular Biosciences, University of Graz,

Heinrichstrasse 31/Humboldtstrasse 50, 8010 Graz, Austria

J. G. Bogner-Strauss

Institute for Genomics and Bioinformatics,

Graz University of Technology, Petersgasse 14,

8010 Graz, Austria phenotype. Our data implicate an as yet unrecognized principle that insufficient lipolysis influences macrophage polarization and actin polymerization, resulting in impaired macrophage migration. Sustained phosphorylation of focal adhesion kinase [due to inactivation of its phosphatase by elevated levels of reactive oxygen species (ROS)] results in defective Cdc42, Rac1 and RhoA activation and in increased and sustained activation of Rac2. Inhibition of ROS production restores the migratory capacity of Atgl-/- macrophages. Since monocyte and macrophage migration are a prerequisite for infiltrating the arterial wall, our results provide a molecular link between lipolysis and the development of atherosclerosis.

Keywords Lipolysis - Small Rho GTPases ·

Adipose triglyceride lipase - Macrophages - Cytoskeleton · Atherosclerosis

\section{Introduction}

Accumulation of macrophages in damaged tissues is a critical process in the pathogenesis of inflammatory diseases such as atherosclerosis. Macrophages are a heterogeneous and phenotypically polarized cell type consisting of classically activated (pro-inflammatory M1) macrophages, which promote strong interleukin (IL)-12-mediated Th1 inflammatory responses, and alternatively activated M2 macrophages with anti-inflammatory properties [1]. Endothelial cells play an important role in the recruitment of inflammatory cells by the expression of chemokines and adhesion molecules, which induce the rolling, adhesion and transmigration of leukocytes into the vascular walls [2]. In addition, inflammatory cytokines enhance the permeability of the endothelial monolayer by altering intercellular 
junctions and cell attachment to the extracellular matrix [3], which leads to activation of tyrosine kinases such as Src kinase and focal adhesion kinase (FAK). FAK is an integrinbinding non-receptor tyrosine kinase, which, on integrin ligation, autophosphorylates its Y397 residue, thereby allowing binding of Src family kinases. The consequence of activated FAK is the phosphorylation of several tyrosine residues of FAK such as Y576 and Y577 [4, 5].

Macrophages navigate along a chemotactic gradient [6]. The basic migratory cycle includes the extension of a protrusion, formation of stable attachments near the leading edge, translocation of the cell body forward, and release of adhesions and retraction at the cell rear [7]. During this wellcoordinated process, the recruitment of macrophages to the site of inflammation depends critically on cytoskeletal rearrangement [8], in particular of actin microfilaments (F-actin), which control membrane plasticity and cell motility. Polymerization of actin drives the initial extension of the plasma membrane at the cell front [9] and regulates cell morphology through contraction and relaxation, thereby generating the mechanical force that is required for moving the cell body forward. The interaction of integrins with the extracellular matrix stabilizes the adhesions by recruiting signaling and cytoskeletal proteins. Activation of FAK at the leading edge results in stimulation of the extracellular signalregulated kinase 2 (ERK2), which promotes matrix proteolysis and facilitates focal contact turnover [10]. Release of adhesions and retraction at the rear completes the migratory cycle allowing net translocation of the cell in the direction of the chemotactic gradient.

Numerous regulators of actin polymerization have been described, most prominently small GTPases of the Rho family and their regulators and effectors [11]. Small GTPases cycle between an inactive GDP-bound and an active GTP-bound state. RhoA, Rac1 and Cdc42, the best characterized members of this protein family, are essential for the organization of the actin cytoskeleton and promote actin structures such as stress fibers (RhoA), lamellipodia (Rac1) and filopodia (Cdc42) [12, 13]. Rho-GTPases participate in the regulation of phagocytosis, cell polarity, proliferation, survival, gene transcription, microtubule dynamics and vesicular transport pathways [11].

We have recently reported on impaired phagocytosis of macrophages lacking adipose triglyceride lipase (ATGL) [14], an enzyme catalyzing the initial step of triacylglycerol (TG) hydrolysis in multiple tissues and cells $[15,16]$ including macrophages. Thus, Atgl-/- macrophages represent a TG-rich foam cell model even in the absence of exogenous lipid loading with unchanged cholesterol concentrations [14]. Transplantation of Atgl-/- bone marrow into an atherosclerotic mouse model resulted in a markedly reduced plaque development [17]. The attenuation of lesion formation in these mice might be due (at least in part) to decreased leukocyte infiltration into the arterial wall. In the present study we investigated whether ATGL modulates the cytoskeleton rearrangement of macrophages and its influence on cell spreading and migration.

\section{Materials and methods}

Animal studies

Atgl-/- and hormone-sensitive lipase (Hsl)-/- mice were generated as described elsewhere [16, 18]. The studies were performed with Atgl-/- mice, $\mathrm{Hsl}-/-$ mice and wild-type (Wt) littermates backcrossed at least seven times on a $\mathrm{C} 57 \mathrm{Bl} / 6$ genetic background. The mice were kept on a standard chow diet (4\% fat and $21 \%$ protein; ssniff, Soest, Germany) and a regular $12 \mathrm{~h}$ dark/light cycle. Animal experiments were performed in accordance with the standards established by the Austrian Federal Ministry of Science and Research, Division of Genetic Engineering and Animal Experiments (Vienna, Austria).

Macrophage isolation and cultivation

Peritoneal macrophages were harvested from the peritoneum of 8 week old female mice 3 days after i.p. injection of $3 \mathrm{ml} \mathrm{3 \%}$ thioglycolate medium. Cells were washed three times with PBS and cultivated in DMEM. Since Atgl-/macrophages cannot mobilize TG and die after prolonged starvation, macrophages were serum-starved for no longer than $3 \mathrm{~h}$. Thereafter the macrophages were incubated in DMEM containing monocyte chemotactic protein-1 (MCP1; $50 \mathrm{ng} / \mathrm{ml}$ ), stromal cell-derived factor-1 (SDF-1; $60 \mathrm{ng}$ / ml) (both from Peprotech, Vienna, Austria) or lysophosphatidic acid (LPA; $9 \mathrm{ng} / \mathrm{ml}$ ) (R\&D Systems, Minneapolis, $\mathrm{MN}$ ) for $0,2,5$ and $10 \mathrm{~min}$. To increase intracellular TG concentrations and to stimulate lipolysis, macrophages from $\mathrm{Wt}$ mice were serum-starved for $12 \mathrm{~h}$ and then incubated with VLDL $(150 \mu \mathrm{g} / \mathrm{ml})$ for $18 \mathrm{~h}$. The increase in lipolysis was determined by measuring glycerol release into the medium in comparison to untreated Wt macrophages using a commercial kit (DiaSys, Flacht, Germany).

Isolation of mouse embryonic fibroblasts (MEFs)

Pregnant female Atgl-/- and Wt mice (12.5-14.5 days post coitum) were killed by cervical dislocation. The uterus with the embryos was removed and placed in 10-cm dishes containing sterile PBS. The head of each embryo from Atgl-/- mice was removed with a fresh razor blade for genotyping since breeding was performed using male Atgl+/- mice. Innards and non-fibroblastic tissues were discarded. After the transfer of each body into a separate 
6-cm dish, the body was minced into small pieces in $1 \mathrm{ml}$ cell culture-grade trypsin-EDTA $(0.25 \%)$. The dishes were placed into an incubator $\left(37^{\circ} \mathrm{C}, 5 \% \mathrm{CO}_{2}\right)$ for $30 \mathrm{~min}$. During the incubation the tissue was disaggregated by pipetting every $10 \mathrm{~min}$. After the addition of $5 \mathrm{ml}$ DMEM, the cell suspension was forced through a syringe equipped with a $20 \mathrm{G}$ needle into a $15-\mathrm{ml}$ tube. The cell suspension was centrifuged for $3 \mathrm{~min}$ at $200 \mathrm{~g}$. The supernatant was discarded, and pelleted cells were subsequently resuspended in $15 \mathrm{ml}$ aMEM. The cell suspension was transferred to $10-\mathrm{cm}$ dishes. The MEFs reached $80 \%$ confluence after $\sim 48 \mathrm{~h}$. Cells from each well were then split onto five 6-cm dishes (passage 1). After reaching $\sim 80 \%$ confluence the MEFs were serum-starved for $12 \mathrm{~h}$, treated with LPA $(10 \mathrm{ng} / \mathrm{ml})$ for indicated time points and then used for pull-down assays.

\section{Cytokine secretion}

Mouse peritoneal macrophages from Wt and Atgl-/- mice were incubated in DMEM/10\% lipoprotein-deficient serum (LPDS) in the absence and presence of MCP-1 $(5 \mathrm{ng} / \mathrm{ml})$ for $24 \mathrm{~h}$. Secretion of IL-6, IL-10 and TGF $\beta$ from the cells into the medium was quantified by ELISA (R\&D Systems, Minneapolis, MN) according to the manufacturer's protocol.

\section{Immunofluorescence}

Macrophages were fixed with $4 \%$ formaldehyde in PBS for $30 \mathrm{~min}$ at room temperature. Cells were permeabilized with $0.5 \%$ Triton X-100 in PBS for 5 min and blocked with $3 \%$ skim milk for 20 min. Cells were incubated with antiRac2 (1:200) and anti-RhoA (1:100) antibodies for $1 \mathrm{~h}$. Thereafter, the cells were stained for $1 \mathrm{~h}$ with anti-rabbit AlexaFluor-488 (1:250) for visualization of Rac2 and RhoA (all antibodies purchased from Molecular Probes, Invitrogen, Vienna, Austria). For F-actin staining cells were blocked with $3 \%$ BSA supplemented with $0.5 \%$ Triton $\mathrm{X}-100$. Then the cells were incubated with phalloidin AlexaFluor-568 (Molecular Probes, Invitrogen, Vienna, Austria) for $25 \mathrm{~min}$. After washing the cells with PBS they were mounted in Vectashield/DAPI (Vector, Burlingame, CA). Images were taken on an Olympus FSX100 fluorescence microscope (Olympus, Hamburg, Germany).

Adhesion and spreading assays

Macrophages from Wt and Atgl-/- mice were plated onto fibronectin-coated coverslips $(25 \mu \mathrm{g} / \mathrm{ml})$ and adhered for $30 \mathrm{~min}$. Thereafter, SDF-1 (40 ng/ml), LPA $(9 \mathrm{ng} / \mathrm{ml})$ and MCP-1 (50 ng/ml) were added for 0, 2, 5 and $10 \mathrm{~min}$. Cells were fixed with $4 \%$ formaldehyde and stained with phalloidin AlexaFluor-568 to visualize F-actin. Spread cells were counted using a Leica SP5 confocal microscope (Leica Microsystem, Vienna, Austria). The surface area of the cells was measured using MetaMorph software (Molecular Devices, Downingtown, PA).

\section{Western blotting}

Mouse peritoneal macrophages were incubated with LPA (9 ng/ml) and SDF-1 (60 ng/ml) for $0,2,5$ and $10 \mathrm{~min}$. Cells were lysed in Ripa buffer $(50 \mathrm{mM}$ Tris- $\mathrm{HCl} \mathrm{pH} 8$, $150 \mathrm{mM} \mathrm{NaCl}, 1 \%$ Triton X-100 and $0.5 \%$ sodium desoxycholate) in the presence of $1 \mu \mathrm{g}$ protease inhibitor cocktail (Sigma-Aldrich, Vienna, Austria). Lysates were separated by SDS-PAGE and transferred onto nitrocellulose protran BA85 membranes (Whatman, Vienna, Austria). Membranes were blocked in $5 \%$ BSA plus $0.1 \%$ Tween-20 for $2 \mathrm{~h}$ and incubated with the following primary rabbit polyclonal antibodies (dilution 1:1,000): pFAK (Tyr576/577), pSrc (Tyr416), p-p38, FAK, Src, p38, Akt, ERK1/2 and mouse anti-pAkt (Ser473), SHP-2, pSHP-2 and pERK1/2 (all purchased from Cell Signaling, Vienna, Austria). All blots were incubated overnight at $4{ }^{\circ} \mathrm{C}$. The horseradish peroxidase-conjugated goat anti-rabbit (1:5,000) (Santa Cruz Biotechnology, Heidelberg, Germany) and rabbit anti-mouse antibodies $(1: 1,000)$ (Dako, Glostrup, Denmark) were visualized by enhanced chemiluminescence detection (ECL, GE Healthcare, Piscataway, NJ) on an AGFA Curix Ultra X-ray film (Siemens, Graz, Austria). For normalization, band intensities were quantified by Image $\mathbf{J}$ software.

\section{Cell migration assays}

For in vivo cell migration, Wt and Atgl-/- mice were injected with $3 \mathrm{ml} \mathrm{3 \%}$ thioglycolate. Peritoneal lavage was isolated $72 \mathrm{~h}$ after injection and F4/80-positive cells were determined on a FACSCalibur flow cytometer (BD Biosciences, San Jose, CA). In vitro cell migration assays of monocytes and macrophages were performed using 24-well transwell plates (pore sizes: 3 and $5 \mu \mathrm{m}$, respectively; Corning, Vienna, Austria). Two million cells were added into the upper well. The lower chamber contained DMEM with and without MCP-1 (50 ng/ml), SDF-1 (60 ng/ml) and LPA $(9 \mathrm{ng} / \mathrm{ml})$. Transwell plates were incubated in $37^{\circ} \mathrm{C}$ for $4 \mathrm{~h}$. For both in vivo and in vitro assays, macrophages were stained with anti-F4/80 antibody (eBioscience, Vienna, Austria). Macrophages and monocytes that had migrated to the bottom wells were enumerated on a FACSCalibur flow cytometer [19]. To evaluate the effect of the antioxidant $N$-acetyl cystein (NAC, $20 \mathrm{mM}$ ) and the NADPH oxidase inhibitor diphenyleneidonium (DPI, $2 \mu \mathrm{M})$ (CalBiochem/EMD 
Bioscience, San Diego, CA) on macrophage migration, cells were pretreated with NAC and DPI for $30 \mathrm{~min}$ before loading into the migration chamber.

\section{Real-time PCR}

Total RNA was isolated from Wt and Atgl-/- macrophages or monocytes using the peqGOLD kit (PeqLab, Vienna, Austria) according to the manufacturer's instructions. Aortas from Wt and Atgl-/mice were isolated, and RNA was extracted using TriFast (PeqLab, Vienna, Austria). Two $\mu \mathrm{g}$ total RNA was reverse transcribed using the High Capacity cDNA Reverse Transcription Kit (Applied Biosystems, Vienna, Austria). Quantitative real-time PCR was performed on an ABI prism 7900 real-time PCR instrument (Applied Biosystems, Vienna, Austria) using the Quantifast Sybr Green PCR kit (Qiagen, Hilden, Germany). Primer sequences are available on request. Data are displayed as expression ratios of target genes normalized to the expression of hypoxanthine-guanine phosphoribosyltransferase (HPRT) for cells and cyclophilin A for aortas as internal reference in each sample. Quantitative real-time PCR data were analyzed by the $2^{-\mathrm{ddCt}}$ method as described [20].

\section{Rho GTPase pull-down assay}

The GST-fusion protein pGEX-T-PAK N containing the p21-activated-kinase domain for Rac1, Rac2 and $\mathrm{Cdc} 42$ (kindly provided by Silvio Gutkind, National Institute of Health, Bethesda, MD) was expressed in E. coli and bound onto glutathione agarose bead. Macrophages grown in $5 \mathrm{~cm}$ dishes were serum-starved for $2 \mathrm{~h}$ in DMEM and stimulated with LPA $(9 \mathrm{ng} / \mathrm{ml})$ for 0, 2, 5 and $10 \mathrm{~min}$. The reaction was stopped by putting the dishes on ice and washing the cells with ice-cold PBS. Cells were lysed with $500 \mathrm{ml}$ lysis buffer $[50 \mathrm{mM}$ Tris- $\mathrm{HCl}, 200 \mathrm{mM} \mathrm{NaCl}$, $10 \mathrm{mM} \mathrm{MgCl} 2,1 \mathrm{mM}$ dithiothreitol (DTT), $1 \mathrm{mM}$ PMSF, $10 \mathrm{mg} / \mathrm{ml}$ aprotinin, $10 \mathrm{mg} / \mathrm{ml}$ leupeptin, $5 \%$ glycerol and $1 \%$ Nonidet P-40] and then centrifuged at $14,000 \mathrm{rpm}$ for $5 \mathrm{~min}$ at $4^{\circ} \mathrm{C}$. The cell lysates were incubated with $30 \mu \mathrm{g}$ of pre-chilled RBD (for RhoA) or p21-activated-kinase (for Rac1, Rac2 and Cdc42) glutathione agarose beads for $30 \mathrm{~min}$ at $4^{\circ} \mathrm{C}$ on a rotary shaker. The cell lysates were used for determination of the total amount of each GTPase. Samples were separated by SDS-PAGE. Proteins were transferred onto polyvinylidene fluoride membranes (Millipore, Billerica, MA) and blocked in TBST buffer [1 mM $\mathrm{CaCl}_{2}, 136 \mathrm{mM} \mathrm{NaCl}, 2.5 \mathrm{mM} \mathrm{KCl}, 25 \mathrm{mM}$ Tris-HCl, $0.1 \%$ (v/v) Tween-20, $\mathrm{pH} 7.4$ ] containing $5 \%$ non-fat dry milk for $1 \mathrm{~h}$. Membranes were incubated with anti-RhoA $(1: 1,000),-\operatorname{Rac} 1(1: 4,000),-\operatorname{Rac} 2(1: 1,000)$ or $-\mathrm{Cdc} 42$ $(1: 1,000)$ antibodies at $4^{\circ} \mathrm{C}$ overnight. After washing, the blots were incubated for $2 \mathrm{~h}$ with either HRP-conjugated goat anti-rabbit $(1: 4,000)$ or HRP-conjugated goat antimouse $(1: 4,000)$ antibodies. Proteins were visualized using Pierce ECL Western Blotting Substrate (Thermo Scientific, Rockford, IL). The band intensities of active GTP-bound GTPase and total GTPase expressions were measured using ImageJ software. Data are expressed as the ratios of GTPGTPase to total GTPase expression. Fold changes were calculated relative to the ratios in unstimulated cells.

\section{ROS production}

Wt and Atgl-/- macrophages were cultivated in black 96-well plates in DMEM in the absence (control) or presence of LPA $(9 \mathrm{ng} / \mathrm{ml})$ or SDF-1 $(60 \mathrm{ng} / \mathrm{ml})$ at $37^{\circ} \mathrm{C}$ for 5 and $10 \mathrm{~min}$, or pretreated with NAC $(20 \mathrm{mM})$ and DPI $(2 \mu \mathrm{M})$ for $30 \mathrm{~min}$. The cells were washed and thereafter incubated with 5-(and-6)-chloromethyl-2',7'-dichlorodihydrofluorescein diacetate, acetyl ester (DCFDA, $50 \mu \mathrm{M}$ ) (Sigma-Aldrich, Vienna, Austria) for $10 \mathrm{~min}$. Intracellular ROS concentrations were determined by the hydrolysis of DCFDA to fluorescent $2^{\prime}, 7^{\prime}$-dichlorofluorescein (excitation: $488 \mathrm{~nm}$; emission: $535 \mathrm{~nm}$ ) in a FlexStation ${ }^{\mathrm{TM}}$ II device (Molecular Devices, Downingtown, PA).

\section{Statistics}

Statistical analyses were performed using GraphPad Prism 5.0 software. The significance was determined by Student's $t$ test. Data with $>2$ groups or $\geq 2$ independent variables were analyzed by ANOVA, followed by the Bonferroni post-hoc test. Data are presented as mean values \pm SEM. Significance levels between Wt and Atgl-/- macrophages were set at $* p<0.05, * * p \leq 0.01, * * * p \leq 0.001$, and at ${ }^{\#} p<0.05,{ }^{\# \#} p \leq 0.01, \quad{ }^{\# \# \#} p \leq 0.001$ between different conditions.

\section{Results}

Atgl-/- macrophages exhibit an anti-inflammatory phenotype

Previous data showed that transplantation of Atgl-/- bone marrow into an atherosclerotic mouse model resulted in reduced MCP-1 serum concentrations [17]. Therefore, we first determined whether ATGL deficiency alters the cytokine expression patterns in macrophages. For that purpose, we measured pro- and anti-inflammatory cytokines in Wt and Atgl-/- macrophages. Production of pro-inflammatory IL-6 was $30 \%$ decreased in Atgl-/compared to $\mathrm{Wt}$ macrophages, whereas the release of the anti-inflammatory cytokines $\mathrm{IL}-10$ and TGF- $\beta$ was 
increased by 1.6- and 3.6-fold, respectively (Fig. 1a). mRNA levels for the M1 marker genes $\mathrm{C}-\mathrm{C}$ chemokine ligand 5 (Ccl5/Rantes) and chemokine ( $\mathrm{C}-\mathrm{X}-\mathrm{C}$ motif) ligand 1 (Grol) were almost absent in Atgl-/- macrophages. In contrast, mRNA expression of antiinflammatory genes such as macrophage chemoattractant protein $2(M c p 2)$, sphingosine kinase $1(S p h k 1)$, C-type lectin domain family 7 member A $($ Clec $7 a)$, mannose receptor $1(\mathrm{Mrc})$ and arginase $1(\mathrm{Arg})$ was increased in macrophages lacking ATGL (Fig. 1b). This pattern of proand anti-inflammatory cytokine expression strongly indicates that $A t g l-/-$ macrophages resemble features of anti-inflammatory M2-like macrophages.

Migration is diminished in Atgl-/- macrophages

Cytokines play important roles in macrophage migration in chemoattractant gradients to the sites of inflammation. Therefore, we examined the effect of ATGL deficiency on macrophage migration in vivo after an i.p. thioglycolate
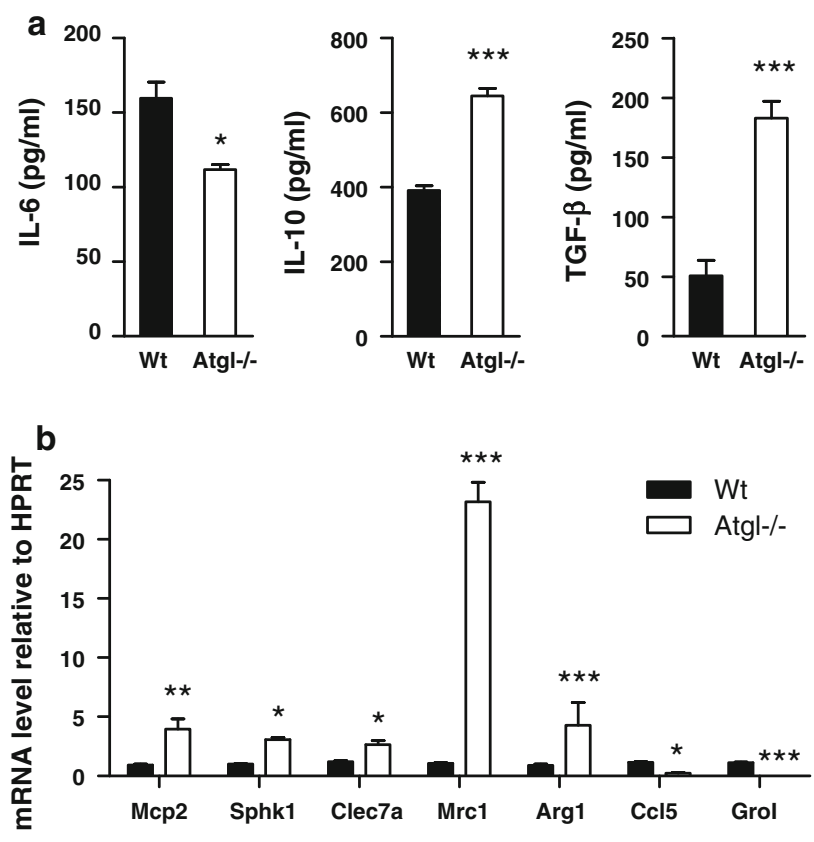

Fig. 1 Atgl-/- macrophages exhibit phenotype of alternatively activated macrophages (a) Wt and Atgl-/- macrophages were incubated in DMEM/10\% LPDS, and cytokine secretion was determined by ELISA. Data represent mean values $(n=6)$ of two independent experiments \pm SEM measured in triplicate. (b) Quantitative real-time PCR analyses of macrophages from Wt and Atgl-/mice; Mcp2, macrophage chemoattractant protein 2; Sphk1, sphingosine kinase 1; Clec7a, C-type lectin domain family 7 member A; Mrc1, mannose receptor 1; Arg1, arginase 1; Ccl5/Rantes, C-C chemokine ligand 5; Gro1, chemokine (C-X-C motif) ligand 1. Data represent mean values $(n=4)$ of two independent experiments \pm SEM performed in triplicate repeats. $* p<0.05, * * p \leq$ $0.01, * * * p \leq 0.001$ injection. After $72 \mathrm{~h}$, macrophage recruitment to the peritoneal cavity of Atgl-/- mice was reduced by $64 \%$ compared to Wt mice (Fig. 2a). These data indicate a critical role for ATGL in macrophage recruitment in peritoneal inflammation. To confirm these observations made in vivo, we next determined the capacity of Atgl-/macrophages to migrate toward various chemoattractants also in vitro. Indeed, migration of Atgl-/- macrophages toward MCP-1, SDF-1 and LPA was markedly reduced in comparison to $\mathrm{Wt}$ macrophages $(56,69$ and $63 \%$, respectively) (Fig. 2b). Since macrophage migration is dependent on the presence of surface adhesion molecules, we determined mRNA levels of key adhesion molecules in macrophages, monocytes and aortas from Atgl-/- mice. mRNA levels of very late antigen 4 (Vla4; ligand for vascular cell adhesion molecule 1) and lymphocyte function-associated antigen 1 (Lfa1; ligand for intercellular adhesion molecule 1) were drastically reduced in Atgl-/compared to Wt macrophages (93 and $88 \%$, respectively) (Fig. 2c). Moreover, E-selectin mRNA levels were reduced by $28 \%$ as well, whereas P-selectin mRNA expression was not significantly changed between Wt and Atgl-/- macrophages. Intercellular adhesion molecule 1 (Icam1) mRNA was downregulated by $84 \%$ in aortas of $A t g l-/-$ mice, whereas vascular cell adhesion protein 1 (Vcam1) mRNA was unchanged (Fig. 2d). Consistent with the major impact of ATGL on surface antigen expression, we observed that also migration of Atgl-/- monocytes toward all three chemoattractants was markedly diminished (supplemental Figure S1a). mRNA levels of Vla4 and P-selectin were decreased by 46 and $37 \%$ in Atgl-/monocytes, whereas Lfa1 and E-selectin mRNA levels were comparable in monocytes of both genotypes (supplemental Figure S1b). In summary, these data demonstrate that ATGL deficiency inhibits monocyte and macrophage migration in vivo and in vitro, which might be due to decreased expression of adhesion molecules.

Reduced actin polymerization and increased cell spreading in Atgl-/- macrophages

The rearrangement of actin filaments is a prerequisite for leukocyte adhesion and migration. We therefore determined the consequences of ATGL deficiency on actin polymerization and cell spreading. Remarkably, Atgl-/macrophages immediately started spreading when plated on fibronectin-coated coverslips, which was not observed with Wt macrophages. Atgl-/- macrophages formed broad protrusions, whereas Wt macrophages remained round-shaped and apparently smaller (Fig. 3a). The number of cells with spread shape was increased by 3.5-fold (Fig. 3b), and the mean cellular area was 1.4fold higher in Atgl-/- compared to Wt macrophages 

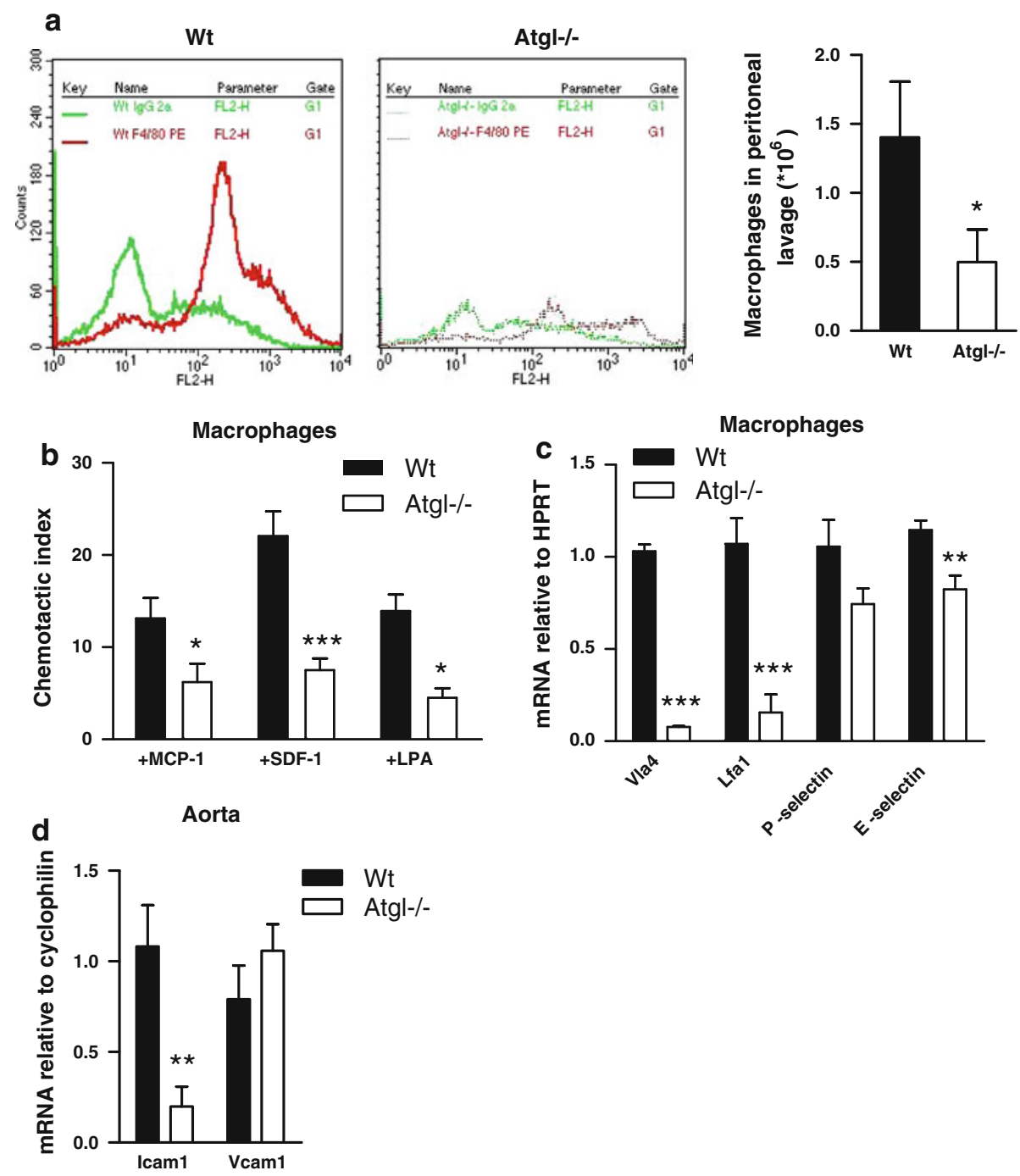

Fig. 2 Reduced migration of Atgl-/- macrophages (a) Wt and Atgl-/- mice were challenged with $3 \%$ thioglycolate by intraperitoneal injection. Peritoneal lavage was collected 3 days after injection, and the macrophage-specific marker F4/80 was measured by flow cytometry. Data represent means $(n=6)$ of three independent experiments. (b) Macrophages from Wt and Atgl-/- mice were added to the upper chamber of transwell plates and were allowed to migrate through the membrane (pore size: $5 \mu \mathrm{m}$ ) into the lower chamber containing DMEM in the absence and presence of MCP-1 $(50 \mathrm{ng} / \mathrm{ml})$, SDF-1 $(60 \mathrm{ng} / \mathrm{ml})$ or LPA $(9 \mathrm{ng} / \mathrm{ml})$ at $37^{\circ} \mathrm{C}$ for $4 \mathrm{~h}$. Migrated cells were counted by flow cytometry. Chemotactic indexes were calculated from the ratio of the cells that had migrated in the

(Fig. 3c). Cell spreading requires actin polymerization to form lamellipodia, and we therefore evaluated whether SDF-1, LPA and MCP-1 affected actin polymerization differently in Wt and Atgl-/- macrophages. After 2 and 5 min of incubation, all three chemoattractants induced the reorganization of actin filaments in Wt cells, which, however, appeared to be impaired in Atgl-/- macrophages (Fig. 3d and supplemental figure S2a). After

presence or absence of the respective chemoattractant. Data of two independent experiments are presented as means $(n \geq 5) \pm$ SEM. c, d Total RNA was isolated from Wt and $\mathrm{Atgl}-/-$ (c) macrophages and (d) aorta, reverse transcribed, and mRNA expression of (c) very late antigen 4 (Vla4), lymphocyte function-associated antigen 1 (Lfa1), P-selectin, E-selectin and (d) vascular cell adhesion protein 1 (Vcam1) and intercellular adhesion molecule 1 (Icam1) was determined by real-time PCR including normalization to (c) HPRT and (d) cyclophilin A. Data are expressed as mean values $(n=6)$ of two independent experiments \pm SEM performed in triplicate repeats. $* p<0.05, * * p \leq 0.01, * * * p \leq 0.001$

5 min of exposure to the chemoattractants, Wt macrophages showed an extending head and a retracting tail with F-actin rich protrusions. In marked contrast, Atgl-/- macrophages had broad lamellopodia in different directions and no apparent leading edge-uropod morphology (Fig. 3d). The percentage of spread cells increased considerably in Atgl-/- macrophages on stimulation with chemoattractants (Fig. $3 \mathrm{e}$ and 
Fig. 3 Accelerated cell spreading in $\mathrm{Atgl}-\mathrm{/}-$ macrophages. a, d Macrophages from Wt and Atgl-/- mice were plated on fibronectincoated glass coverslips for $10 \mathrm{~min}(\mathbf{a})$ in the absence or (d) presence of SDF-1 $(60 \mathrm{ng} / \mathrm{ml})$ and LPA $(9 \mathrm{ng} / \mathrm{ml})$ for 2 and 5 min. Adherent cells were fixed and stained with phalloidin AlexaFluor-586. Images were taken using a Leica AP5 AOBS confocal microscope. Arrow in (a) points to broad protrusions in an Atgl-/- macrophage. Scale bars $5 \mu \mathrm{m}$. b, e Cell spreading was calculated by counting the percentage of spread cells. c, f Mean cellular area was measured using MetaMorph software. Data from two independent experiments are presented as mean values of $>650$ cells per genotype \pm SEM. g Fluorescence intensity of phalloidin AlexaFluor-586stained Wt and Atgl-/macrophages was measured by flow cytometry. The histograms show untreated cells (brown) and macrophages treated with LPA for 2 min (green), 5 min (yellow) and $10 \mathrm{~min}$ (blue). $* p<0.05, * * p \leq 0.01$, $* * * p \leq 0.001$
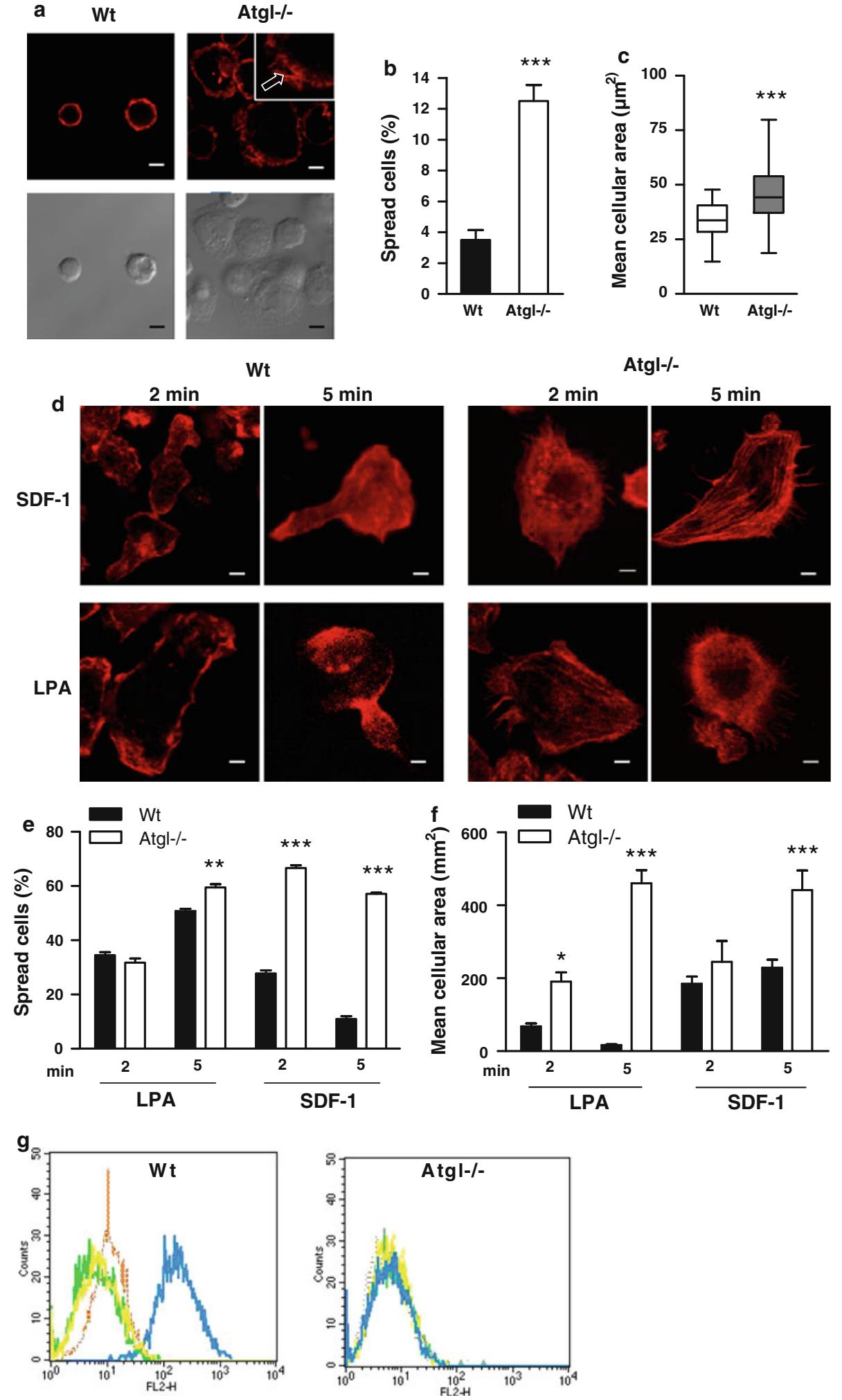

supplemental Figure S2b), resulting in a significantly increased mean cellular area of these cells (Fig. 3f and supplemental Figure S2c). This different spreading and migration behavior after LPA treatment is due to defective actin polymerization in Atgl-/- macrophages, as evidenced by flow cytometry (Fig. 3g). 

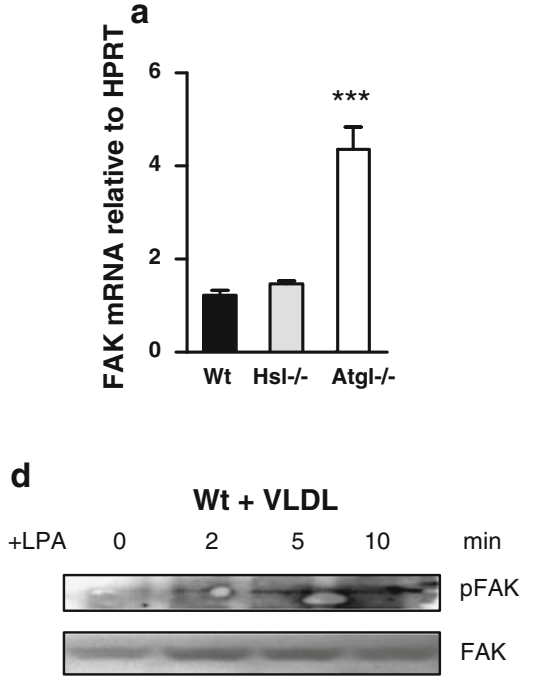

f

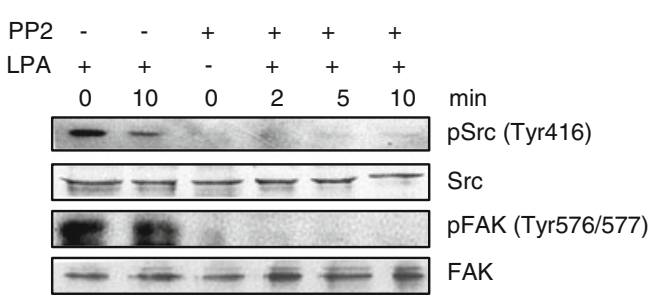

h

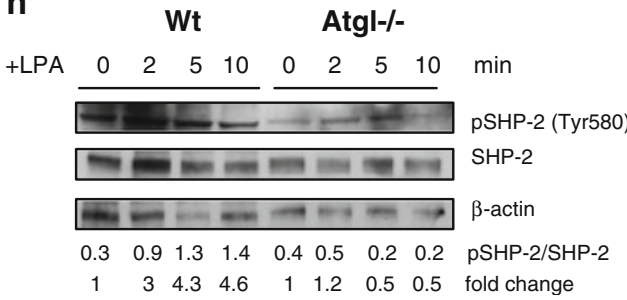

C

e b Wt Hsl-/- Atgl-/-
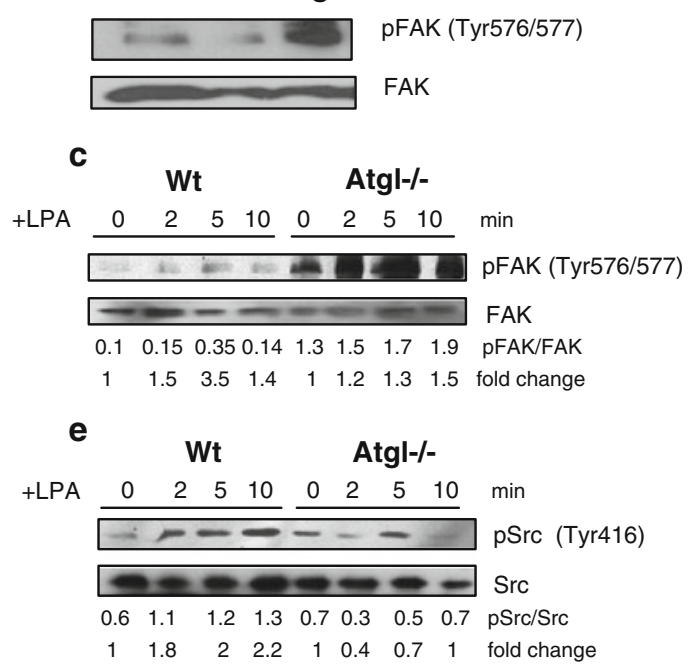

g

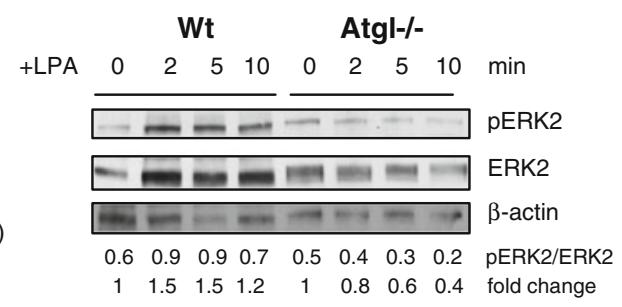

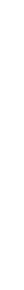

Fig. 4 Sustained FAK phosphorylation mediates spreading in Atgl-/- macrophages in a Src kinase-dependent manner. a Total RNA was isolated from Wt, $\mathrm{Hsl}-/-$ and Atgl-/- macrophages, reverse transcribed and mRNA expression of FAK was determined by real-time PCR including normalization to hypoxanthine guanine phosphoribosyl transferase (HPRT). Data represent mean values $(n=4)$ of two independent experiments \pm SEM performed in triplicate repeats. ${ }^{* *} p \leq 0.001$. b Western blot analysis in macrophage lysates from Wt, $\mathrm{Hsl}-/-$ and $\mathrm{Atgl}-/-$ mice using antibodies specific for total and phosphorylated (p)FAK (Tyr576/577). c, e Wt, Atgl-/- and (d) Wt macrophages loaded with VLDL $(150 \mu \mathrm{g} / \mathrm{ml}$ for $18 \mathrm{~h}$ ) were incubated with LPA $(9 \mathrm{ng} / \mathrm{ml})$ for $0,2,5$ and $10 \mathrm{~min}$. The

Sustained activation of FAK in $A t g l-/-$ macrophages due to inactivation of its phosphatase

To identify the pathways that are responsible for the enhanced cell spreading of Atgl-/- macrophages, we performed real-time PCR for FAK, which plays an important role in integrin-mediated cell adhesion [21], spreading and focal adhesion formation [22]. In Atgl-/macrophages, FAK expression was 3.5-fold upregulated lysates were subjected to Western blot analysis using antibodies specific for (c, d) total and pFAK and (e) total and pSrc (Tyr416). f $\mathrm{Atgl}-\mathrm{/}-$ macrophages were preincubated with the Src kinase inhibitor 4-amino-5-(4-chlorophenyl)-7-( $t$-butyl) pyrazolo [3,4-d] pyrimidine (PP2, $10 \mu \mathrm{M})$ for $30 \mathrm{~min}$ and then exposed to SDF-1 $(60 \mathrm{ng} / \mathrm{ml})$ for $0,2,5$ and $10 \mathrm{~min}$. Western blot analysis was performed using the above-mentioned antibodies. $\mathbf{g}$, h The lysates were subjected to Western blot analysis using antibodies specific for (g) total and pERK2 and (h) total and pSHP-2 (Tyr580). Ratios of two Western blots were calculated by dividing band intensities of phosphorylated to total protein expression. Fold changes were calculated relative to the ratios of unstimulated cells

compared to Wt cells (Fig. 4a). To investigate whether this alteration was specific for ATGL deficiency, we determined FAK mRNA expression in $\mathrm{Hsl}-\mathrm{l}-$ macrophages. HSL is responsible for the second step in lipolysis by hydrolyzing diacylglycerol more efficiently than TG, thereby inhibiting lipolysis independent of ATGL. As shown in Fig. 4a, FAK expression was comparable in $\mathrm{Hsl}-\mathrm{/}-$ and $\mathrm{Wt}$ macrophages. Immunoblotting revealed that only a very small portion of FAK was activated in $\mathrm{Wt}$ 
and $\mathrm{Hsl}-\mathrm{/}-$ macrophages (Fig. 4b), whereas hyperphosphorylation of FAK was detectable in Atgl-/macrophages. Phosphorylated FAK was transiently increased after $5 \mathrm{~min}$ and decreased to the basal level after a 10-min treatment of Wt macrophages with LPA and SDF-1 (Fig. 4c and supplemental Figure S3a). In contrast, FAK remained activated even after sustained conditions in Atgl-/- macrophages (Fig. 4c and supplemental Figure $\mathrm{S} 3 \mathrm{a})$. To assess whether stimulated lipolysis affected FAK phosphorylation, we serum-starved $\mathrm{Wt}$ macrophages for $12 \mathrm{~h}$ and then promoted lipolysis by incubating the cells with VLDL $(150 \mu \mathrm{g} / \mathrm{ml})$ for $18 \mathrm{~h}$. Stimulation of lipolysis was confirmed by measuring glycerol release into the medium (data not shown). Loading of Wt macrophages with VLDL resulted in a moderate time-dependent increase in FAK phosphorylation (Fig. 4d).

Since tyrosine phosphorylation of FAK is regulated by Src family kinases [23], we next determined activation of Src in Wt and Atgl-/- macrophages. Whereas activated Src was gradually increased in $\mathrm{Wt}$ macrophages after stimulation with LPA and SDF-1 for 2, 5 and $10 \mathrm{~min}$, it was significantly reduced in Atgl-/- macrophages (Fig. 4e and supplemental figure S3b). To assess whether FAK itself acts as a Src kinase substrate, we pretreated Atgl-/- macrophages with the Src kinase inhibitor 4-amino-5-(4-chlorophenyl)-7-( $t$-butyl) pyrazolo [3, 4-d] pyrimidine (PP2) for $30 \mathrm{~min}$. As expected, inhibition of Src kinase was associated with blunted phosphorylation of Src in the absence and presence of LPA or SDF-1 (Fig. 4f and supplemental figure S3c). In addition, Src kinase inhibition also resulted in impaired FAK activation confirming that phosphorylation of FAK in Atgl-/- macrophages occurs in a Src kinase-dependent manner. Notably, we observed a drastically reduced ERK2 activation in Atgl-/- macrophages on treatment with LPA (Fig. 4g), which may explain the defect in focal contact turnover in these cells.

The sustained phosphorylation of FAK in Atgl-/macrophages prompted us to investigate the role of tyrosine phosphatase SHP-2 as the major regulator of FAK phosphorylation dynamics [24]. Phosphorylation of SHP-2 at Tyr580 releases its autoinhibition, thereby stimulating its phosphatase activity [25]. In contrast to gradually increased phosphorylation of SHP-2 in Wt macrophages, we found reduced phosphorylation of SHP-2 in Atgl-/- macrophages in response to LPA (Fig. 4h). These findings indicate that sustained phosphorylation of FAK is due to inactivated SHP-2 in Atgl-/- macrophages.

Defective Cdc42, Rac1 and Rho signaling in Atgl-/- macrophages

To investigate the mechanism of defective actin polymerization and cell polarization in $A t g l-/-$ macrophages, we analyzed the activation of Rho GTPase proteins by pulldown assays. Activated GTP-bound Cdc42 remained stable in Wt macrophages for up to 5 min after treatment with LPA, but dropped to almost undetectable levels after $10 \mathrm{~min}$ of treatment (Fig. 5a). In contrast, Cdc42 activation in Atgl-/macrophages was markedly decreased compared to Wt cells both under treated and untreated conditions (Fig. 5a). Whereas the level of activated Rac1 was comparable in $\mathrm{Wt}$ and Atgl-/- macrophages in the absence of LPA (Fig. 5b), Rac1 was induced after 2 min of LPA treatment in $\mathrm{Wt}$ macrophages. The induction of Rac1, however, was significantly delayed in Atgl-/- macrophages and was not observed until 10 min after LPA addition. LPA treatment of VLDL-loaded Wt macrophages transiently reduced GTPbound $\mathrm{Cdc} 42$, which returned to baseline levels after $10 \mathrm{~min}$ of LPA treatment (supplemental Figure S4). The level of GTP-Rac1 in VLDL-loaded Wt macrophages was equivalent to Wt and Atgl-/- macrophages in the absence of LPA (supplemental Figure S4). The delayed Rac1 activation after LPA treatment was comparable to Atgl-/- macrophages, indicating that the impaired Rac 1 induction is a consequence of intracellular TG accumulation.

To test whether the alterations observed in Atgl-/macrophages are specific for this cell type or occur also in other cells we isolated MEFs from Atgl-/- and Wt mice and determined FAK phosphorylation as well as Rac1 and Cdc42 activation in these cells. Since activation of Rac1 was highest after 30 min in Wt MEFs (data not shown), we incubated the cells for 0 and 30 min with LPA. Comparable with macrophages, FAK phosphorylation was significantly higher in Atgl-/- than in Wt MEFS both in the absence and presence of LPA (Fig. 5c). In accordance with FAK activation, GTPbound Rac1 and $\mathrm{Cdc} 42$ were drastically reduced in the absence of ATGL even after 30 min of LPA treatment, when compared to MEFs from Wt mice (Fig. 5c).

Next we determined the activation and translocation of RhoA. In Wt macrophages, RhoA activation was increased by LPA stimulation for 2 and $5 \mathrm{~min}$, whereas the treatment of Atgl-/- macrophages with LPA drastically reduced GTP-bound RhoA expression (Fig. 5d). In Wt and Atgl-/- macrophages, RhoA was predominantly localized around the nucleus in the absence of any chemoattractant (Fig. 5e). After the addition of LPA or SDF-1, RhoA translocated to the leading edge and rear of $\mathrm{Wt}$ cells. In contrast, RhoA remained localized close to the nucleus in Atgl-/- macrophages even in the presence of LPA or SDF-1 (Fig. 5e).

Sustained Rac2 activation and increased ROS production in Atgl-/- macrophages

Since Rac2 also plays a critical role in regulating chemotaxis (at least in neutrophils), we next determined Rac2 
Fig. 5 Defective Cdc42, Rac1 and RhoA activation in Atgl-/macrophages. a, b, d Wt and Atgl-/- macrophages and (c) MEFs were serum-starved for $3 \mathrm{~h}$ and then incubated in DMEM containing $9 \mathrm{ng} / \mathrm{ml}$ LPA. Cell lysates were incubated with glutathionesepharose beads complexed with GST-PBD fusion proteins to pull down GTP-bound Rac1, Cdc42 and RhoA. Precipitates were resolved by SDS-PAGE in parallel with whole cell lysates (total Cdc42/Rac1/RhoA).

Protein expression of $(\mathbf{a}, \mathbf{c})$ Cdc42 and GTP-Cdc42, (b, c) Rac1 and GTP-Rac1, (d) RhoA and GTP-RhoA was determined by Western blot analysis using specific antibodies. Band intensities were quantified using Image $\mathbf{J}$ software, and ratios of two Western blots of activated to total protein expression were determined. Fold changes were calculated relative to the ratios in unstimulated cells. e Wt and Atgl-/- macrophages were plated on fibronectin-coated coverslips and serum-starved for $2 \mathrm{~h}$. Cells were incubated in the absence (control) and presence of SDF-1 $(60 \mathrm{ng} / \mathrm{ml})$ and LPA $(9 \mathrm{ng} / \mathrm{ml})$ for $10 \mathrm{~min}$ at $37^{\circ} \mathrm{C}$. Thereafter, cells were fixed and stained with antiRhoA and AlexaFluor-586 phalloidin to visualize F-actin. Pictures were recorded using an Olympus FSX100 fluorescence microscope. Scale bars $5 \mu \mathrm{m}$

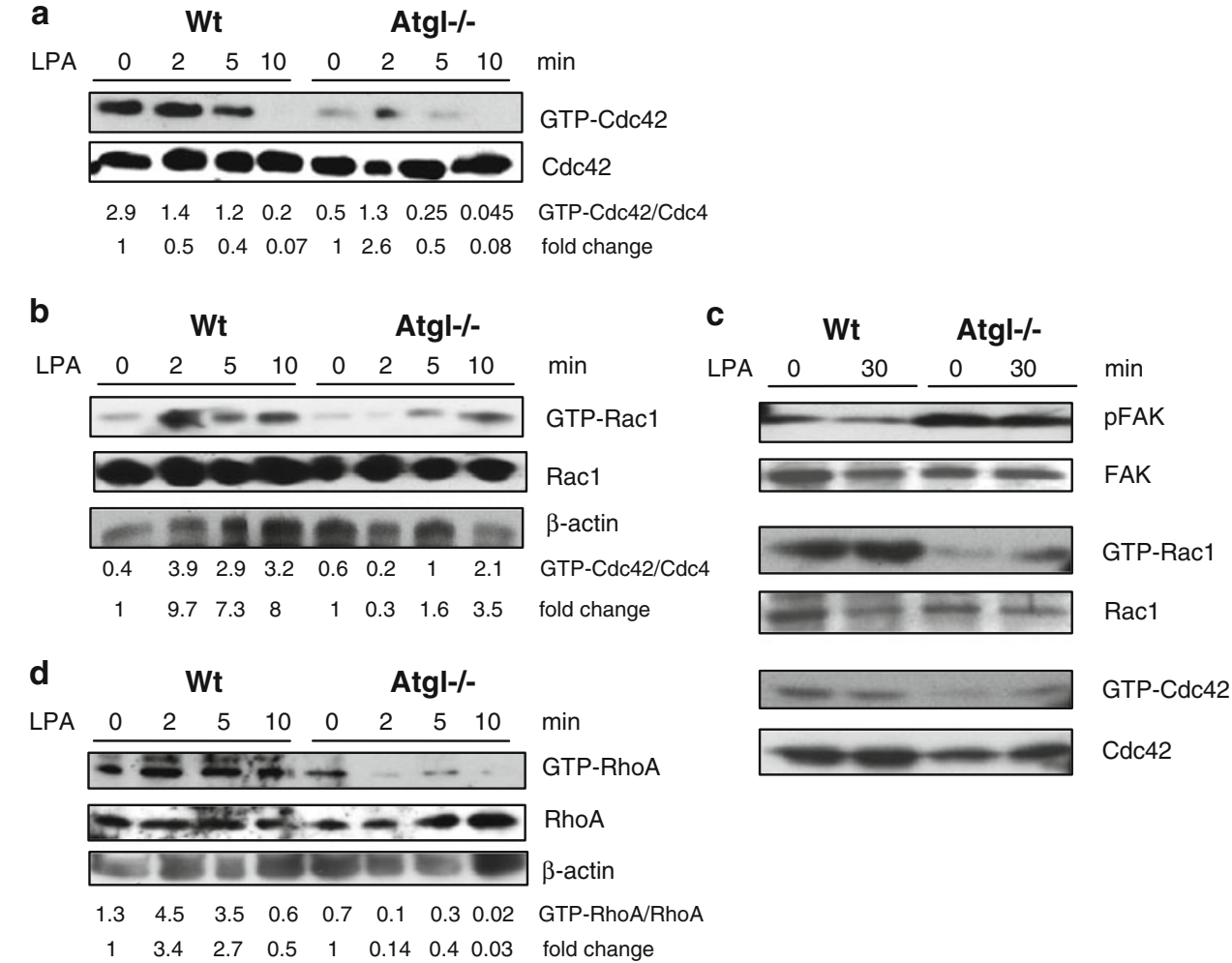

e

Wt

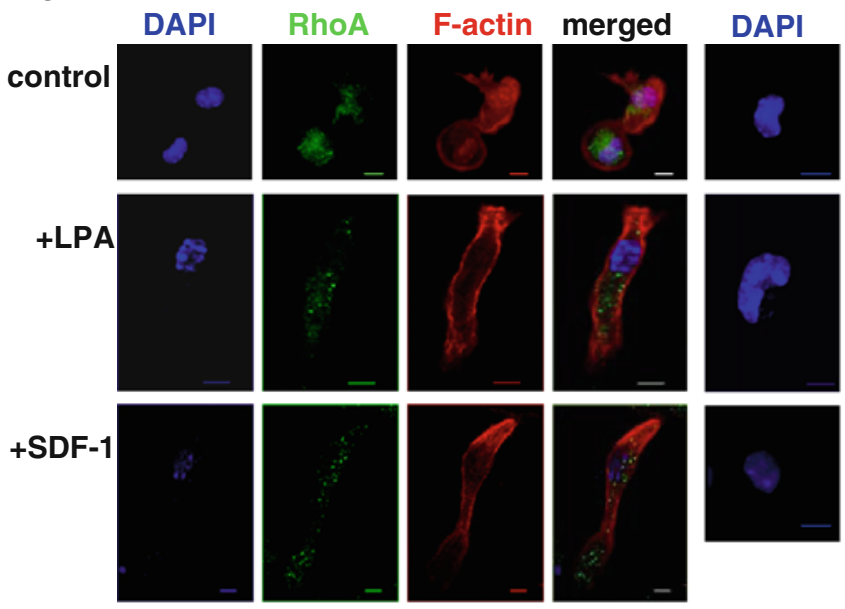

Atgl-/-

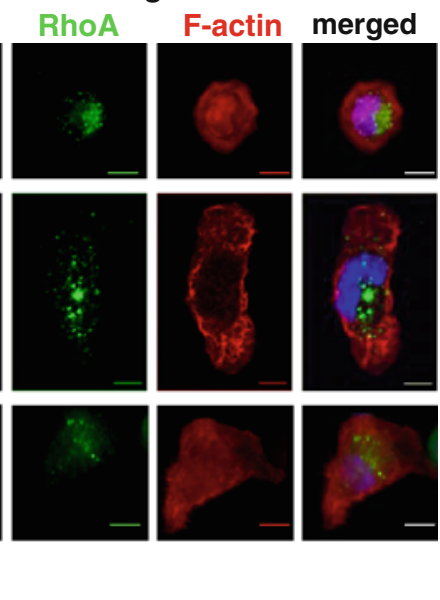

activation in $\mathrm{Wt}$ and Atgl-/- macrophages. Activated Rac2 was present in unstimulated Wt macrophages, and increased by the addition of LPA after 5 min but quickly turned over and was almost completely absent after $10 \mathrm{~min}$ of treatment (Fig. 6a). In Atgl-/- macrophages, we observed sustained Rac2 activation, which even further increased after $10 \mathrm{~min}$ of LPA stimulation (Fig. 6a). Immunofluorescence revealed that (similar to RhoA) Rac2 was situated near the nucleus in Wt macrophages lacking colocalization with F-actin (Fig. 6b). In contrast, Rac2 was found in the cytosol and near the cell periphery in Atgl-/macrophages, and colocalized with F-actin in these cells.

GTP-Rac2 regulates NADPH oxidase activator, an enzyme catalyzing the NADPH-dependent one electron reduction of $\mathrm{O}_{2}$ to superoxide anion $\left(\mathrm{O}_{2}^{-}\right)$. Hence, we examined ROS production in $\mathrm{Wt}$ and Atgl-/- macrophages as monitored by the conversion of DCFDA to fluorescent DCF. Consistent with a sustained activation of Rac2 in Atgl-/- macrophages, we found highly increased intracellular ROS concentrations in these cells (Fig. 6c). 


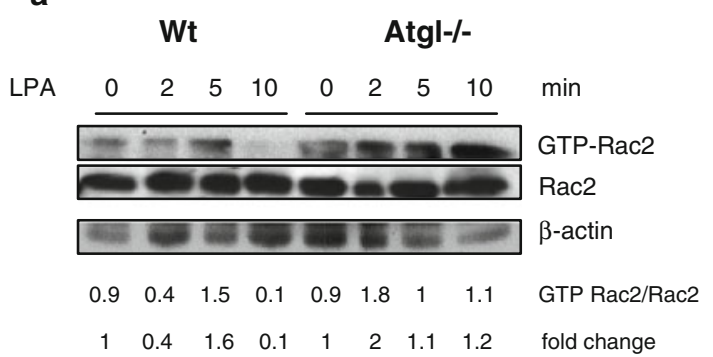

b

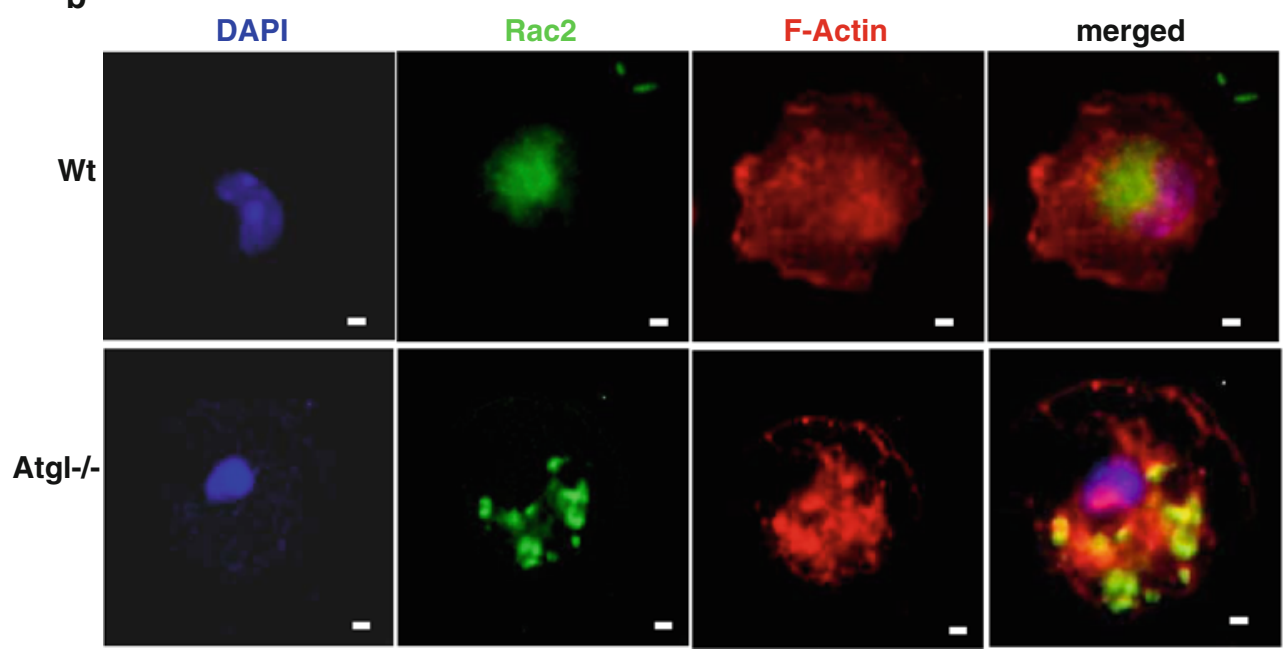

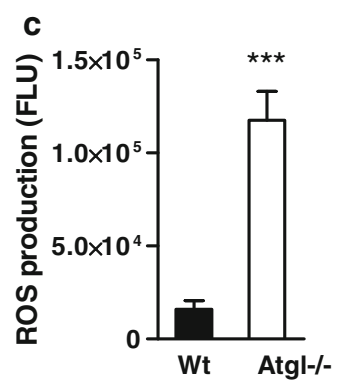

Fig. 6 Increased ROS production and Rac2 activation in Atgl-/macrophages. a Wt and Atgl-/- macrophages were incubated in DMEM containing LPA $(9 \mathrm{ng} / \mathrm{ml})$ for $0,2,5$ and $10 \mathrm{~min}$. Cell lysates were incubated with glutathione-sepharose beads complexed with GST-PBD fusion protein to pull down GTP-Rac2. Precipitates were resolved by SDS-PAGE in parallel with whole cell lysates (total Rac2). Band intensities of two Western blots were quantified using Image $\mathbf{J}$ software, and ratios of activated to total protein expression were determined. b Wt and Atgl-/- macrophages were plated on

Inhibition of ROS production in Atgl-/- macrophages eliminates sustained phosphorylation of FAK and defective cell migration

Finally, to investigate whether increased ROS production in Atgl-/- macrophages is responsible for their altered migration behavior, we used NAC as an anti-oxidant and DPI as an NADPH oxidase inhibitor. Atgl-/- macrophages pre-incubated with NAC or DPI showed a $72 \%$ fibronectin-coated coverslips for $10 \mathrm{~min}$, fixed and stained with antiRac2 antibody and phalloidin AlexaFluor-586. Pictures were taken on an Olympus FSX100 fluorescence microscope. Scale bars $5 \mu \mathrm{m}$. $\mathbf{c ~ W t}$ and Atgl-/- macrophages were loaded with 5-(and-6)-chloromethyl$2^{\prime}, 7^{\prime}$-dichlorodihydrofluorescein diacetate, acetyl ester (DCFDA, $50 \mu \mathrm{M}$ ) for $10 \mathrm{~min}$. Fluorescence was measured in a FlexStation device (excitation: $488 \mathrm{~nm}$; emission: $535 \mathrm{~nm}$ ). Data are presented as means $(n=3) \quad \pm$ SEM measured in quadruplicate repeats. $* * * p \leq 0.001$

inhibition of ROS concentration (Fig. 7a). Since ROS formation inactivates SHP-2 (Meng et al., 2002), we examined whether the activation of FAK in Atgl-/macrophages could be reduced by pre-incubating the cells with NAC and DPI. In fact, DPI and NAC prevented the sustained activation and restored the transient phosphorylation of FAK in response to LPA (Fig. 7b). To further analyze whether reduced FAK phosphorylation affected migration, we incubated Atgl-/- macrophages for $10 \mathrm{~min}$ 


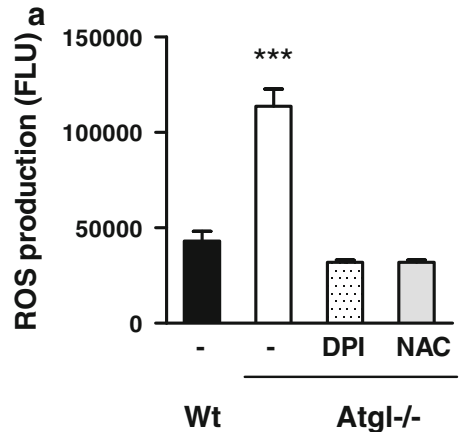

b
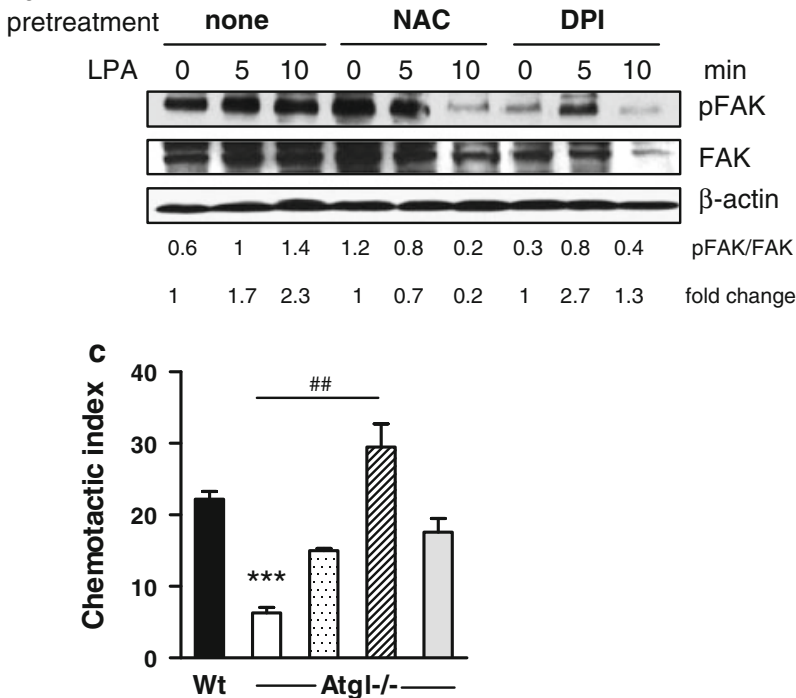

$\mathrm{DPI}(\mu \mathrm{M}) \quad-\quad-0.22$ -

Fig. 7 Intracellular ROS production inhibits migration by sustained FAK phosphorylation. a Atgl-/- macrophages were pretreated with $N$-acetyl-cystein (NAC) $(20 \mathrm{mM})$ and diphenyleneidonium (DPI) $(2 \mu \mathrm{M})$ for $30 \mathrm{~min}$. Intracellular ROS production was determined by hydrolysis of DCFDA $(50 \mu \mathrm{M})$ to fluorescent DCF in a FlexStation. Data represent mean values $(n=5) \pm$ SEM of two independent experiments measured in quadruplicate repeats. b Atgl-/- macrophages were pretreated with NAC $(20 \mathrm{mM})$ and DPI $(2 \mu \mathrm{M})$ for $30 \mathrm{~min}$ and then stimulated with LPA for 5 and $10 \mathrm{~min}$. Cells were lysed and subjected to Western blotting to detect total and phosphorylated FAK. Representative blots from two independent experiments are shown. Band intensities were quantified using Image $\mathbf{J}$ software and ratios of activated to total protein expression were determined. Fold changes were calculated relative to the ratios in unstimulated cells. c Atgl-/- macrophages were pretreated with NAC $(20 \mathrm{mM})$ and DPI $(2 \mu \mathrm{M})$ for $30 \mathrm{~min}$, and then chemotaxis toward LPA $(9 \mathrm{ng} / \mathrm{ml})$ was determined. Data represent mean values $(n=4) \pm$ SEM of two independent experiments performed in duplicate repeats. $* * * p \leq 0.001 ;{ }^{\# \#} p \leq 0.01$

with DPI and NAC and determined their chemotaxis toward LPA. As shown in Fig. 7c, migration of Atgl-/macrophages was completely restored to the levels in $\mathrm{Wt}$ cells, using the same concentrations of NAC and DPI that were required to generate dephosphorylated FAK (Fig. 7c). Taken together, these data indicate that elevated ROS production in Atgl-/- macrophages leads to sustained FAK activation, which impairs cell adhesion and migration.

\section{Discussion}

Macrophages are versatile cells that fulfill various functions in response to environmental changes. Macrophagederived foam cells play important roles in the progression of inflammatory diseases like atherosclerosis. We have recently reported that Atgl-/- macrophages, which accumulate TG-rich lipid droplets, show impaired phagocytosis because of a reduced availability of free fatty acids as energy substrate [14]. Moreover, we found markedly decreased atherosclerosis in low-density lipoprotein receptor $(\mathrm{Ldlr})-/$ - mice after transplantation of Atgl-/bone marrow compared to $\mathrm{Wt}$ bone marrow-transplanted $L d l r-/-$ mice [17]. The attenuation of lesion formation in these mice might be due (at least in part) to decreased leukocyte infiltration into the arterial wall. Recruitment of monocytes and macrophages to the site of inflammation and the secretion of cytokines are critical steps in the inflammatory response and necessitates cytoskeleton rearrangements. In this study we show that the migratory capacity of Atgl-/- monocytes and macrophages is markedly reduced in vivo and in vitro. Furthermore, our data provide evidence that the decreased chemotaxis of Atgl-/- macrophages is a consequence of reduced expression of adhesion molecules and defective actin dynamics.

For active chemotaxis, actin polymerization and depolymerization are a prerequisite. To accomplish efficient migration, leukocytes (including macrophages) extend a front F-actin-rich lamellipodium constituting the leading edge, and the uropod, in which both the microtubule and intermediate-filament network are retracted during migration [6]. Thus, cell migration needs actin polymerization, which drives lamellipodia extension, disruption of existing focal contacts and formation of new contacts [26]. We therefore hypothesize that defective actin polymerization in Atgl-/ - macrophages is the cause of reduced cell migration.

In migrating cells, dephosphorylation of FAK is temporally associated with disruption of focal adhesions and increased motility [27, 28]. Atgl-/- macrophages, however, show increased and sustained phosphorylation of FAK, suggesting that hyperphosphorylation of FAK is one of the factors involved in impaired migration and enhanced spreading. These alterations are likely specific for ATGL deficiency and the concomitant increase in intracellular TG concentrations, since the lack of HSL, an enzyme more involved in the second step in lipolysis (by catalyzing the 
hydrolysis of diacylglycerol) than in TG mobilization, fails to induce FAK phosphorylation. Incubation of Wt macrophages with VLDL results in moderate FAK phosphorylation. This might be the consequence of two independent features of VLDL loading: first, VLDL promotes lipolysis, thereby reducing intracellular TG concentrations. Second, the concentration of VLDL we used results in TG accumulation comparable to Atgl-/macrophages [29], indicating that ATGL activity might be (at least partly) inhibited by VLDL. FAK-Src activation and signaling to ERK2 promote FAK release from existing focal contacts, thereby triggering FAK re-binding and activation at different focal contacts in a migrating cell [10]. We therefore conclude that impaired activation of SHP-2, the major phosphatase for FAK, and decreased ERK2 activation are involved in sustained FAK phosphorylation of Atgl-/- macrophages. Direct phosphorylation of FAK by Src kinase family members is an important step in the formation of an active FAK signaling complex [23]. Consequently, inhibition of Src abolished FAK phosphorylation in Atgl-/- macrophages, implicating that Src is a substrate for FAK also in these cells. A regulated balance between cytoskeletal assembly and disassembly, which has to be maintained to ensure cell movement, is impeded in Atgl-/- macrophages.

The signaling pathways required for actin polymerization, which are associated with FAK phosphorylation, include Rho GTPases (in particular RhoA, Rac1 and Cdc42) and their regulator and effector proteins [11]. Cdc42 acts at the front of the macrophage to control the cell's orientation toward an extracellular stimulus resulting in head extension and tail retraction and eventually in the activation of Rac1 [13, 30]. RhoA regulates the adhesion required for normal tail retraction during leukocytes migration. In Atgl-/- macrophages, RhoA activation is almost abolished in the presence of chemoattractants. RhoA localizes close to the nucleus in Atgl-/- macrophages and fails to translocate to the side or rear on chemoattractant treatment. Activated RhoA is distributed predominantly at the sides and rear in polarized neutrophils [31], which we also observed in $\mathrm{Wt}$ macrophages. We therefore conclude that impaired RhoA activation diminishes RhoA translocation from the nucleus resulting in a severe defect in macrophage polarization. Since Rho activity must be tightly regulated to allow efficient cell migration [30], this defect in cell polarization due to the inability of RhoA to stimulate contractility likely contributes to impaired chemotaxis in Atgl-/- macrophages. By stimulating the formation of filopodia, Cdc42 plays a crucial role in gradient detection and cell polarization. Inhibition of $\mathrm{Cdc} 42$ and Rac1 prevents macrophage polarization and directional movements toward chemoattractants. Cdc42 inhibition renders macrophages to random movement instead of directional migration, whereas inhibition of Rac1 blocks cell movement [30, 32]. In line with the reduced motility of Atgl-/- macrophages, we observed a marked reduction of activated GTP-bound Cdc42 and Rac1 in Atgl-/- macrophages in response to LPA. This effect was not restricted to macrophages because we observed decreased activation of Rac1 and Cdc42 (along with hyperphosphorylation of FAK) also in MEFs lacking ATGL. In macrophages, it is important to note that the chemokine $\mathrm{Ccl} 5 /$ Rantes was shown to induce lamellipodia formation [33] and to activate Rac1 [34]. As pro-inflammatory cytokines, Ccl5/Rantes and Gro-1 play an active role in recruiting leukocytes into inflammatory sites. In Atgl-/- macrophages, Ccl5/Rantes and Gro1 mRNA levels were almost absent. The impact of Gro1 on small Rho GTPase activation has not been investigated so far. Therefore, we hypothesize that together with the lack of RhoA activation and translocation, impaired Cdc42 and Rac1 activation and reduced chemokine expression account for the reduced migratory capacity and in vivo accumulation of $A t g l-/-$ macrophages.

Oxidative inactivation of tyrosine phosphatases such as SHP-2 is induced by intracellular ROS production [35]. The production of ROS through the multiprotein enzyme complex NADPH oxidase is crucial for the immune response. ROS accumulation in macrophages leads to sustained activation of FAK, inactivation of SHP-2 and fast spreading [28]. To address the impact of ROS on FAK activation, we determined ROS concentrations, which were drastically increased in Atgl-/- compared to Wt macrophages in the absence of chemoattractants. Inhibition of ROS production in Atgl-/- macrophages by NAC and DPI triggered the loss of sustained FAK phosphorylation. As a consequence, the phenotype of defective migration of Atgl-/- macrophages was eliminated. Rac2 regulates NADPH oxidase activator and ROS concentrations in phagocytes, most importantly in neutrophils [36]. In macrophages, Rac1 protein expression is $\sim 4$-fold higher compared to Rac2 [37]. A role of Rac2 in macrophage function, however, was demonstrated in Rac2-/- macrophages, which exhibit defective NADPH oxidase activation resulting in reduced ROS formation [37]. Activated Rac2 expression was increased in Atgl-/- macrophages, and we observed Rac2 localization in the cytosol and near the cell periphery, whereas in Wt macrophages Rac2 was localized in and around the nucleus. This finding indicates that Rac2, which is activated in Atgl-/- macrophages, translocates to the membrane as has previously been observed during NADPH oxidase activation in stimulated neutrophils. In these cells, the translocation of Rac2 to the membrane during NADPH oxidase activation exhibits kinetics and stoichiometries consistent with a role of Rac2 in NADPH oxidase regulation [38]. Rac1 also contributes to NADPH 
oxidase activation in macrophages [39]. Since Rac1 activation was rather decreased than increased, we hypothesize that the sustained activation of FAK in Atgl-/- macrophages results from increased ROS concentrations because of activated Rac2.

Data from the present study provide strong evidence that ATGL deficiency leads to an increased secretion of antiinflammatory cytokines accompanied by the development of M2-like macrophages. We conclude that reduced atherosclerosis in $\mathrm{Ldlr}-/ \mathrm{-}$ mice transplanted with $\mathrm{Atgl}-\mathrm{/}-$ bone marrow [17] might be due to altered cytoskeletal dynamics, reduced trafficking and a shift from pro- to antiinflammatory cytokine secretion from the macrophages. Defective activation of the Rho small GTPases RhoA, Cdc42 and Rac1 in Atgl-/- macrophages causes disturbances in actin polymerization and cell polarization. In addition, activation of Rac2 in Atgl-/ - macrophages leads to increased ROS production and impaired dephosphorylation of FAK, resulting in enhanced spreading and impaired migration. Our findings implicate macrophage ATGL as a promising target to reduce inflammation and macrophage infiltration and thereby attenuate atherosclerosis.

Acknowledgments This work was supported by the Austrian Science Fund FWF (SFB-LIPOTOX F30, P19186 and P22521) and the Austrian Federal Ministry of Science and Research (GEN-AU project Genomics of Lipid-associated Disorders-GOLD). E.A., N.A.B.B. and V.K. were funded by the PhD program Molecular Medicine of the Medical University of Graz. The authors thank A. Ibovnik for excellent technical assistance and I. Hindler for mouse care.

Open Access This article is distributed under the terms of the Creative Commons Attribution Noncommercial License which permits any noncommercial use, distribution, and reproduction in any medium, provided the original author(s) and source are credited.

\section{References}

1. Martinez FO, Sica A, Mantovani A, Locati M (2008) Macrophage activation and polarization. Front Biosci 13:453-461

2. Sprague AH, Khalil RA (2009) Inflammatory cytokines in vascular dysfunction and vascular disease. Biochem Pharmacol 78:539-552

3. Campos SB, Ashworth SL, Wean S et al (2009) Cytokineinduced F-actin reorganization in endothelial cells involves RhoA activation. Am J Physiol Renal Physiol 296:F487-F495

4. Parsons JT (2003) Focal adhesion kinase: the first ten years. J Cell Sci 116:1409-1416

5. Brown MC, Cary LA, Jamieson JS, Cooper JA, Turner CE (2005) Src and FAK kinases cooperate to phosphorylate paxillin kinase linker, stimulate its focal adhesion localization, and regulate cell spreading and protrusiveness. Mol Biol Cell 16:4316-4328

6. Vicente-Manzanares M, Sanchez-Madrid F (2004) Role of the cytoskeleton during leukocyte responses. Nat Rev Immunol $4: 110-122$
7. Lauffenburger DA, Horwitz AF (1996) Cell migration: a physically integrated molecular process. Cell 84:359-369

8. Kim JY, Lee YG, Kim MY et al (2010) Src-mediated regulation of inflammatory responses by actin polymerization. Biochem Pharmacol 79:431-443

9. Carson M, Weber A, Zigmond SH (1986) An actin-nucleating activity in polymorphonuclear leukocytes is modulated by chemotactic peptides. J Cell Biol 103:2707-2714

10. Mitra SK, Hanson DA, Schlaepfer DD (2005) Focal adhesion kinase: in command and control of cell motility. Nat Rev Mol Cell Biol 6:56-68

11. Etienne-Manneville S, Hall A (2002) Rho GTPases in cell biology. Nature 420:629-635

12. Ridley AJ (2001) Rho proteins, PI 3-kinases, and monocyte/ macrophage motility. FEBS Lett 498:168-171

13. Nobes CD, Hall A (1995) Rho, rac, and cdc42 GTPases regulate the assembly of multimolecular focal complexes associated with actin stress fibers, lamellipodia, and filopodia. Cell 81:53-62

14. Chandak PG, Radovic B, Aflaki E et al (2010) Efficient phagocytosis requires triacylglycerol hydrolysis by adipose triglyceride lipase. J Biol Chem 285:20192-20201

15. Zimmermann R, Strauss JG, Haemmerle G et al (2004) Fat mobilization in adipose tissue is promoted by adipose triglyceride lipase. Science 306:1383-1386

16. Haemmerle G, Lass A, Zimmermann R et al (2006) Defective lipolysis and altered energy metabolism in mice lacking adipose triglyceride lipase. Science 312:734-737

17. Lammers B, Chandak PG, Aflaki E et al (2011) Macrophage adipose triglyceride lipase deficiency attenuates atherosclerotic lesion development in low-density lipoprotein receptor knockout mice. Arterioscler Thromb Vasc Biol 31:67-73

18. Haemmerle G, Zimmermann R, Hayn M et al (2002) Hormonesensitive lipase deficiency in mice causes diglyceride accumulation in adipose tissue, muscle, and testis. J Biol Chem 277:4806-4815

19. Schratl P, Sturm EM, Royer JF et al (2006) Hierarchy of eosinophil chemoattractants: role of p38 mitogen-activated protein kinase. Eur J Immunol 36:2401-2409

20. Livak KJ, Schmittgen TD (2001) Analysis of relative gene expression data using real-time quantitative PCR and the 2(-Delta Delta C(T)) method. Methods 25:402-408

21. Cary LA, Guan JL (1999) Focal adhesion kinase in integrinmediated signaling. Front Biosci 4:D102-D113

22. Rovida E, Lugli B, Barbetti V et al (2005) Focal adhesion kinase is redistributed to focal complexes and mediates cell spreading in macrophages in response to M-CSF. Biol Chem 386:919-929

23. Calalb MB, Polte TR, Hanks SK (1995) Tyrosine phosphorylation of focal adhesion kinase at sites in the catalytic domain regulates kinase activity: a role for Src family kinases. Mol Cell Biol 15:954-963

24. Yu DH, Qu CK, Henegariu O, Lu X, Feng GS (1998) Proteintyrosine phosphatase Shp-2 regulates cell spreading, migration, and focal adhesion. J Biol Chem 273:21125-21131

25. Lu W, Gong D, Bar-Sagi D, Cole PA (2001) Site-specific incorporation of a phosphotyrosine mimetic reveals a role for tyrosine phosphorylation of SHP-2 in cell signaling. Mol Cell 8:759-769

26. Stossel TP (1994) The machinery of cell crawling. Sci Am 271:54-63

27. Matsumoto K, Nakamura T, Kramer RH (1994) Hepatocyte growth factor/scatter factor induces tyrosine phosphorylation of focal adhesion kinase (p125FAK) and promotes migration and invasion by oral squamous cell carcinoma cells. J Biol Chem 269:31807-31813

28. Park YM, Febbraio M, Silverstein RL (2009) CD36 modulates migration of mouse and human macrophages in response to 
oxidized LDL and may contribute to macrophage trapping in the arterial intima. J Clin Invest 119:136-145

29. Aflaki E, Radovic B, Chandak PG et al (2011) Triacylglycerol accumulation activates the mitochondrial apoptosis pathway in macrophages. J Biol Chem 286:7418-7428

30. Allen WE, Zicha D, Ridley AJ, Jones GE (1998) A role for Cdc42 in macrophage chemotaxis. J Cell Biol 141:1147-1157

31. Xu J, Wang F, Van Keymeulen A et al (2003) Divergent signals and cytoskeletal assemblies regulate self-organizing polarity in neutrophils. Cell 114:201-214

32. Jones GE, Allen WE, Ridley AJ (1998) The Rho GTPases in macrophage motility and chemotaxis. Cell Adhes Commun 6:237-245

33. Di Marzio P, Dai WW, Franchin G et al (2005) Role of Rho family GTPases in CCR1- and CCR5-induced actin reorganization in macrophages. Biochem Biophys Res Commun 331:909-916

34. Weiss-Haljiti C, Pasquali C, Ji $\mathrm{H}$ et al (2004) Involvement of phosphoinositide 3-kinase gamma, Rac, and PAK signaling in chemokine-induced macrophage migration. J Biol Chem 279:43273-43284

35. Meng TC, Fukada T, Tonks NK (2002) Reversible oxidation and inactivation of protein tyrosine phosphatases in vivo. Mol Cell 9:387-399

36. Bokoch GM, Zhao $\mathrm{T}$ (2006) Regulation of the phagocyte NADPH oxidase by Rac GTPase. Antioxid Redox Signal 8:1533-1548

37. Yamauchi A, Kim C, Li S et al (2004) Rac2-deficient murine macrophages have selective defects in superoxide production and phagocytosis of opsonized particles. J Immunol 173:5971-5979

38. Knaus UG, Heyworth PG, Evans T, Curnutte JT, Bokoch GM (1991) Regulation of phagocyte oxygen radical production by the GTP-binding protein Rac 2. Science 254:1512-1515

39. Miyano K, Koga H, Minakami R, Sumimoto H (2009) The insert region of the Rac GTPases is dispensable for activation of superoxide-producing NADPH oxidases. Biochem J 422:373-382 